Thorax (1973), 28, 66.

\title{
Diaphragmatic eventration in infants
}

\author{
F. PAR ÍS, E. B L A S C O, A. CANTÓ, V. T A R A Z ONA, \\ and M. CASILLAS
}

Thoracic Surgery Service, Department of Surgery, Centro Hospitalario 'La Fè', Valencia 9, Spain

\begin{abstract}
Nine cases of diaphragmatic eventration are presented in infants, seven of whom required surgery. All were seen during the first 19 months of life and seven were less than 1 year old. Although differing from other studies, our cases show a predominance of right-sided lesions. Respiratory symptoms were present in most cases, and digestive symptoms appeared in a lesser number of cases. The radiological changes were pronounced in seven of the nine cases, the dome of the diaphragm reaching to the fourth to fifth intercostal spaces. Regarding surgical technique, the authors favour diaphragmatic plication through the thorax. This gave excellent clinical and radiological results and was without mortality or complications.
\end{abstract}

The first description of this anomaly was Petit's (1790) necropsy finding. He described it as a special form of thoracic hernia 'without any separation of the muscular and tendinous fibres in the diaphragm'. The condition was called diaphragmatic eventration by Beclard and Cruveilhier (1829) and this term became generally accepted instead of such names as relaxation, phrenic insufficiency, or hypertrophied diaphragm.

The difference between eventration and hernia is recorded by Bisgard (1947), who describes eventration as 'an abnormally high, or elevated position of one leaf of the diaphragm as a result of paralysis, aplasia, or atrophy of varying degree of the muscle fibres. Its unbroken continuity differentiates it from diaphragmatic hernia'.

Until recently eventration in infants was rarely diagnosed and treated, and descriptions of the condition were mainly from necropsies (BayneJones, 1916; Reed and Borden, 1935). Nowadays this condition is encountered more frequently in paediatric practice. Proof of this is the work of Richard et al. (1959), who collected 80 cases from the literature and added 10 of their own, and the recent work of Monereo, Cortes, and Dominguez (1970) who report 15 cases.

\section{CASE REPORTS}

CASE 1 D.T., a 5-month-old girl, was born at full term and weighed $2 \cdot 8 \mathrm{~kg}$. Delivery was difficult with a breech presentation and artificial respiration was required. The mother stated that the child had had a persistent cough from the first few days after birth until the time of our examination, and that recently her condition had deteriorated with dyspnoea and poor appetite. Her weight was $5.3 \mathrm{~kg}(2.7$ standard deviaw tions below the normal mean weight). Examinatiom disclosed asymmetry of the chest wall with bulgine of the lower right hemithorax and a scaphoig abdomen. Auscultation revealed wheezing and a pecth liar flapping sound in the mid line.

The chest radiograph showed an elevated righ diaphragmatic dome, reaching the level of the fifth rif posteriorly, and a marked shift of the mediastinur to the left. On fluoroscopy the movement of the right diaphragm was paradoxical, and the mediastinum moved to the left on inspiration.

Conservative treatment was tried without succes? the cough, dyspnoea and auscultatory findings persis ing. In view of this, and as there was concern regard ing the infant's development, surgery was reconf mended. A diaphragmatic plication was performe through a thoracic approach and the dome of the diaphragm was lowered to the level of the tenth ri posteriorly. Convalescence was satisfactory and a\$ respiratory symptoms disappeared. After six years the child's development is normal, the mediastinum centrac and the diaphragmatic movement normal.

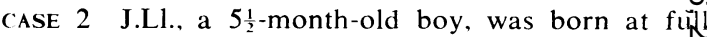
term and weighed $3.0 \mathrm{~kg}$. Delivery was normal, wi cephalic presentation, but respiratory assistance was required. The parents stated that the infant he suffered from repeated bronchopneumonic attacks since birth, accompanied by fever, dyspnoea, an cyanosis.

On clinical examination the infant was under nourished, showing prominence of the lower right chest wall and diminished breath sounds at the rig base. His weight was $5 \mathrm{~kg} \mathrm{(3.4} \mathrm{standard} \mathrm{deviation}$ below the normal mean weight). The chest film show elevation of the right diaphragm, the vertex reachirus the fourth intercostal space. On fluoroscopy the moves ment of the affected side had 'the dicrotic-like patterQ' 
described by Chin and Lynn (1956), with the lax portion moving initially in a paradoxical manner and afterwards normal contraction of the periphery. This produced mediastinal displacement on respiration, which at first sight appeared as authentic paradoxical movement of the right diaphragm.

A diagnosis of right diaphragmatic eventration was made. Plication was performed, lowering the muscle to the ninth rib. Postoperative recovery was satisfactory and after three years the child is progressing well. The position and movement of the diaphragm are normal. There has been no recurrence of the pneumonic episodes.

CASE 3 J.O., a 10 mon:h-old boy, was born by normal delivery with cephalic presentation and weighed $3.2 \mathrm{~kg}$. The child attended hospital because of a chest deformity which was similar to that of cases 1 and 2 . At that time his weight was normal $(8.5 \mathrm{~kg})$. The films showed the right diaphragm to be elevated to the level of the sixth rib with a mediastinal shift to the left. On screening there was a double phase movement of the diaphragm. A plication was performed and three years later the child is well and the chest film is normal.

CASE 4 V.F., an 11-month-old boy. was born wîhout difficulty and weighed $3.5 \mathrm{~kg}$. Presentation was cephalic. Three months after birth he suffered a right bronchopneumonia which produced serious dyspnoea. After that he had repeated acute pneumonic attacks. His weight was $8.0 \mathrm{~kg}$. Physical examination disclosed a bulging of the right lower thoracic cage and a scaphoid abdomen. On fluoroscopy the movement of the diaphragm appeared as in the previous cases, with a slight contraction of the periphery and a relaxation of the middle portion of the right diaphragm. A plication was performed via the thorax with satisfactory results. After one year the patient is in good condition and movement of the diaphragm is normal. There has been no recurrence of the acute bronchopneumonic attacks.

CASE 5 M.D., a 19-month-old girl, was born at full term with cephalic presentation and weighed $3.5 \mathrm{~kg}$. The mother knew of no respiratory symptoms, but since the age of 3 months the girl had suffered from anorexia and vomiting and normal development was retarded. At the time of examination her weight was a normal $10 \mathrm{~kg}$. Examination revealed the same chest wall asymmetry as in the previous cases and the radiograph showed the vertex of the dome reaching the level of the fourth rib. Fluoroscopy revealed sluggish movement of the diaphragm, but it lacked the double phase mobility. In the lateral view, an opaque wedgeshaped zone appeared in front of the dome, which we interpreted as pulmonary atelectasis by compression (Fig. 1).

In addition to the apparent redundancy of the diaphragm, another operative finding was an intralobar sequestration in the anterior segment of the right lower lobe, with a blood supply from infradiaphragmatic vessels. Because of this it was extremely difficult to separate the lung from the diaphragm. In this case the muscle was more atrophic than in the rest of the series, particularly in the anterior and central portions where the peritoneum and the pleura appeared to meet. A right lower lobectomy was performed followed by a plication, which because of the lung resection was not as extensive as in the other cases. Eighteen months after operation the girl is in excellent condition. The position of the diaphragm, although somewhat more elevated than in the rest of the series, conserves good mobility.

CASE 6 R.O., a 12-month-old boy, had a normal birth with cephalic presentation and weighed $3.6 \mathrm{~kg}$. The mother noticed a marked lack of appetite during
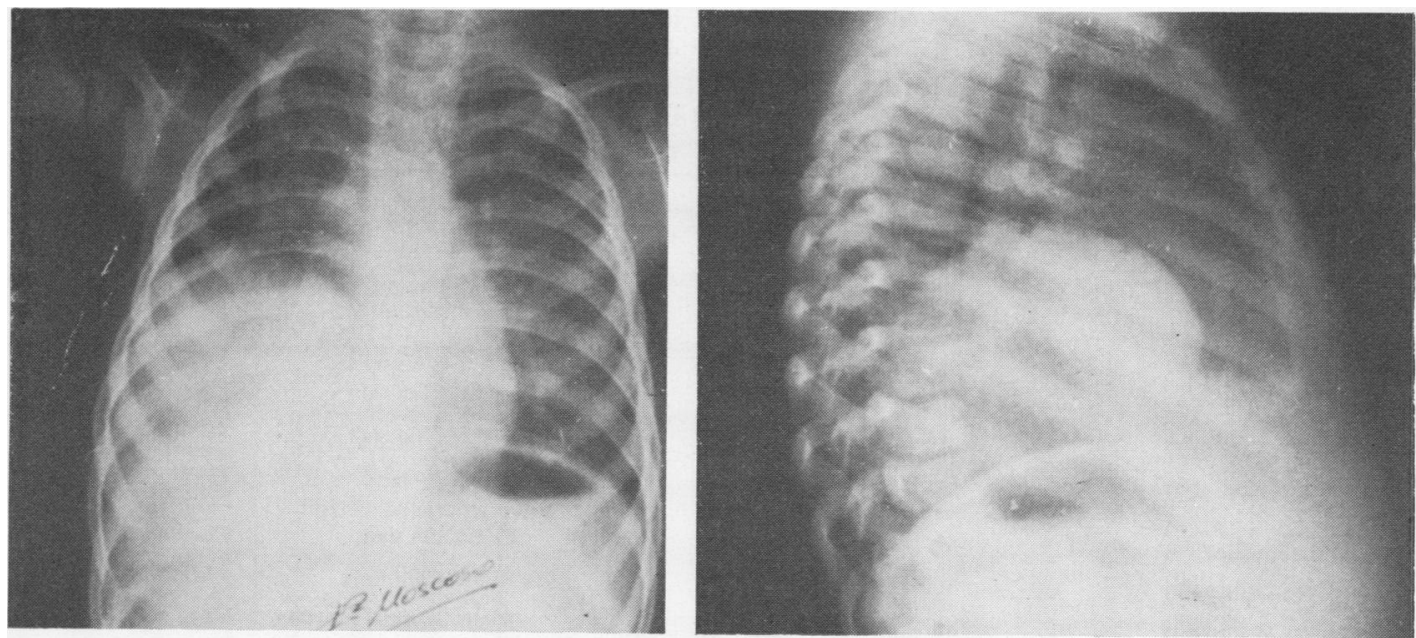

FIG. 1. Anteroposterior and lateral chest films of case 5. The lateral projection reveals an opaque zone in front of the relaxed dome of the diaphragm. 
artificial feeding (Eledon), the child consuming only half or one-third of the normal portion. In addition he suffered from sporadic vomiting and constant constipation. At the age of 5 months he was ill with acute bronchopneumonia and was treated with various antibiotics and corticosteroids. Physical examination revealed gross maldevelopment with a weight of 6.4 kg (3 standard deviations below the normal mean weight). There was marked pigeon's breast and a left Harrington's groove, and prominence of the lower costal margin below. The abdomen was scaphoid. Radiographs revealed elevation of the left dome to the fourth rib space. Both stomach and colon, covered by the relaxed diaphragm, were displaced upwards. On fluoroscopy the dicrotic-like movement of the diaphragm, as previously described, was observed. A plication was performed and the diaphragm was lowered to the eighth intercostal space. The postoperative period was complicated by paralytic ileus with abdominal distension, which resolved in 48 hours. One year after surgery the scaphoid abdomen had disappeared and the weight curve is normal. Radiography shows a central mediastinum and normal movement of the diaphragm.

CASE 7 C.T., a 7-month-old boy, was born at full term with cephalic presentation and weighed $4.0 \mathrm{~kg}$. The mother stated that nine days after birth he had pneumonia with cyanosis. The fever reached $39^{\circ} \mathrm{C}$ $\left(102 \cdot 2^{\circ} \mathrm{F}\right)$ and required antibiotics during a 15-day period. He then had repeated colds with cough and mucoid sputum and low-grade fever from $37.5^{\circ}$ to $38^{\circ} \mathrm{C}\left(99.5^{\circ}\right.$ to $\left.100.4^{\circ} \mathrm{F}\right)$. The findings on physical examination were similar to those in the previous cases and his weight $(7.5 \mathrm{~kg})$ was within normal limits. Radiographs revealed the dome reaching the fourth intercostal space. On screening, the diaphragm had the characteristic dynamics of eventration. A very hypoplastic lower right lobe was discovered during the operation. A plication was performed.
A small muscular wedge of diaphragm removed for examination revealed a degenerative myopatl demonstrating dissociation of the striated muscular fibres included within dense fibroconnective tissue. TFe involuted nature of the muscle fibres was shown the loss of striation, granular transformation of tfoe sarcoplasm, and a marked proliferation of the nucleus.

The boy made a good recovery and the hypoplastic lobe readily expanded. Nine months after operation pulmonary development is normal, and the previog hypoplasia of the lobe is not noticeable.

CASE 8 R.M., a 10-month-old boy, had a norma birth with cephalic presentation and weighed $4.3 \mathrm{~kg}$ At the age of 3 months the mother noticed that the boy's lips had become somewhat cyanotic withoof accompanying cough or fever. Radiographs reveale a shadow in the base of the right lung and because of this we were consulted. A symmetrical thoracic cage was observed and in this he differed from the other patients. His weight was a normal $9 \mathrm{~kg}$. In th films the diaphragm was seen to be markedly relaxe although on fluoroscopy it contracted well and there was no sign of mediastinal herniation. In view of this it was not felt that surgery was indicated, and treat ment was conservative.

CASE 9 A.M., a 15-month-old girl, was born pre maturely at 8 months; she weighed $2 \cdot 0 \mathrm{~kg}$ and requireతe incubation for one and half months until a 3.0 weight was reached. Presentation was cephalic. The was no history of respiratory or digestive symptoms and weight development was normal. The mother wa concerned mainly about the deformity of the thorax and for this reason we were consulted. A bulge in the posterior aspect of the base of the right hemithora. and an anterolateral depression at the level of the sixth and seventh ribs were found on clinical examina tion. Chest films revealed an elevated dome, and the relaxation was more noticeable posteriorly. On screerio ing there was no sign of mediastinal movement an $\overrightarrow{\underline{Q}}$. movement of the diaphragm was normal. Surgery was

T A B L E

CLINICAL DATA OF THE NINE CASES

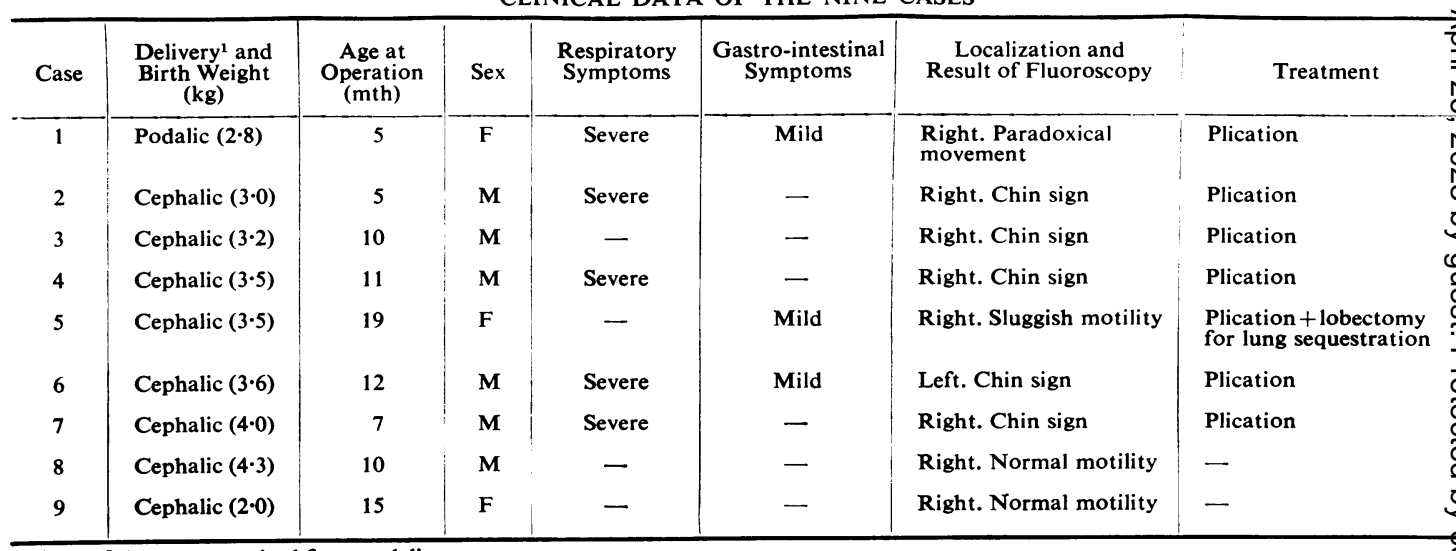

1 None of the cases required forceps delivery 
not considered necessary and observation of the patient was advised.

\section{DISCUSSION}

Diaphragmatic eventration in infants is not a clinical rarity. Beck and Motsay (1952) found that in 2,500 cases of newborn infants $4 \%$ had minor relaxations and $1 \cdot 2 \%$ had serious eventrations. We have had the opportunity of studying nine cases over a six-year period; all of them were brought to us before the age of 19 months and seven of them were less than 1 year old. Two-thirds of the cases were male.

In the Table the most important clinical data are summarized, and in Fig. 2 the pre- and postoperative radiographs of the seven surgical cases are reproduced. The sample indicates a higher frequency of eventration on the right side, which is contrary to the findings in other studies. In the review of Richard et al. (1959), 57 cases of eventration were left-sided, 21 right-sided, and 2 were bilateral. Six of his own cases belonged to the first group and four to the second. In the series of Monereo et al. (1970) there is a slight predominance of left-sided lesions.

An explanation for the higher frequency of right eventration in our series may be the fact that we are thoracic surgeons. The viscus which enters the thorax in right eventration and pushes against the relaxed diaphragm is the liver, and being such a bulky organ it produces greater respiratory difficulty. Also, on occasions we are consulted regarding the possible diagnosis of chest tumours or hydatid cysts which may present diagnostic difficulties in infancy. On the other hand, the stomach and colon are the organs involved on the left side and therefore the diagnosis is made with less difficulty. For this reason, leftsided cases are referred more often to paediatric surgeons.

The relaxation of the diaphragm in all cases requiring surgery was complete, and it was possible to follow the diaphragmatic contour; thus there were no diagnostic problems. In partial eventration confusion is possible. Bonham-Carter, Waterston, and Aberdeen (1962) gave little consideration to the difference between hernia and eventration. Christensen (1959) points out the diffculty of preoperative diagnosis of both conditions. Histological examination of the muscular fibres should give definitive proof.

In practice it is necessary to differentiate between complete and partial eventration. The first is a clinical entity different from hernia and is generally associated with obvious respiratory symptoms. The second, as Richard et al. (1959) point out, acts as a hernial sac regardless of its histology.

According to its aetiology, the diaphragmatic eventration can be either congenital or acquired. The first may be present in the infantile or adult form and the second arises from a temporary or permanent disorder.

The anomaly is based on a defect of penetration of the cervical myotomes in the elements which make up the diaphragm, the septum transversum, the pleuroperitoneal folds, the common mesentery, and the lateral wall of the coelom. Beck and Motsay (1952) consider this disease to be a form of muscular aplasia. They base their argument favouring the congenital theory on the lack of inflammation or degeneration of the phrenic nerve. Bayne-Jones (1916) supports the congenital theory. In our series we have, in two cases, found an associated congenital lesion, a pronounced lobar hypoplasia in one case, and a pulmonary sequestration in another.

We have classified the acquired forms of eventration as those produced by (1) lesions of the phrenic nerve, (2) central neurological disease, (3) myopathies, and (4) changes in diaphragmatic levels caused by neighbouring lesions (pleural effusion, subphrenic abscess, and space-filling abdominal lesions).

In the infantile form, it is necessary to call attention to the lesion of the phrenic nerve caused during delivery, a mechanism that acquired special significance in the work of Bingham (1954), Richard et al. (1957), and Jaubert de Beaujeu and Mollard (1965). The diaphragmatic lesion is usually found to be accompanied by an Erb brachial paralysis, although it can also appear alone. It is necessary to consider stretching of the nerve roots which may occur during the expulsion period of delivery. Breech presentation and forceps delivery favour nerve damage.

We described one of our cases (case 1) as a post-delivery paralytic eventration without brachial plexus palsy. The reasons which led us to consider it thus were (1) a difficult delivery with breech presentation, (2) the appearance during the first few minutes of life of symptoms requiring respiratory resuscitation, and (3) the discovery, during thoracotomy, of an elevated but apparently normal muscular diaphragm. We should say, however, that we lack the evidence to confirm post-delivery nerve damage. The principal argument against this aetiology is the rapid postoperative recovery of the mobility of the diaphragm, which would be unlikely if it was secondary to phrenic nerve damage. 

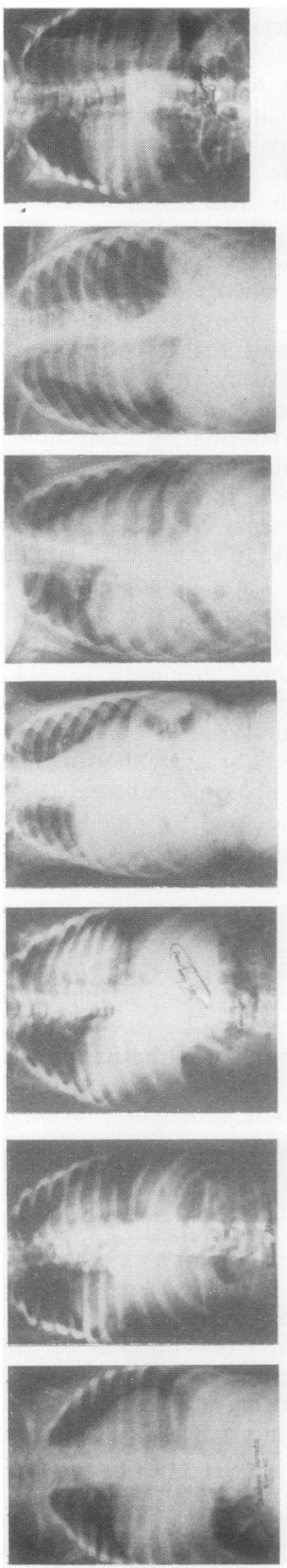

$<$
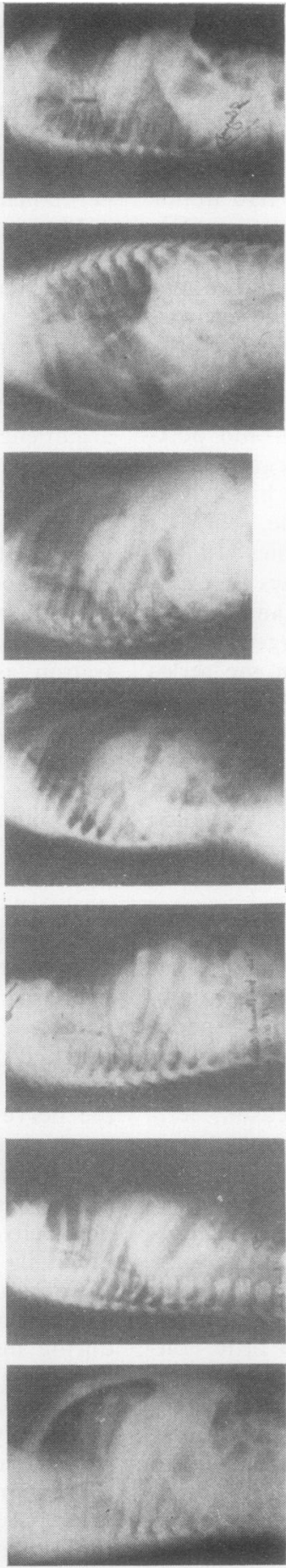

$\infty$
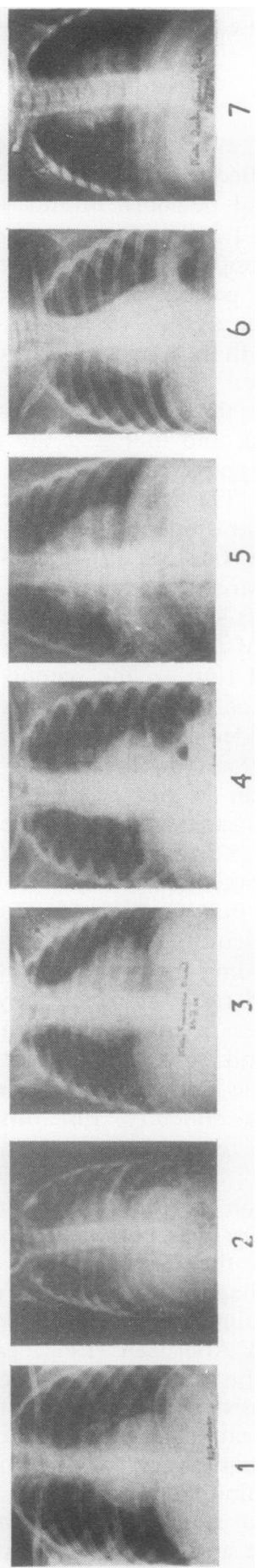

$u$

$\sqrt{-1}$

$\ddot{\breve{s}}$ 

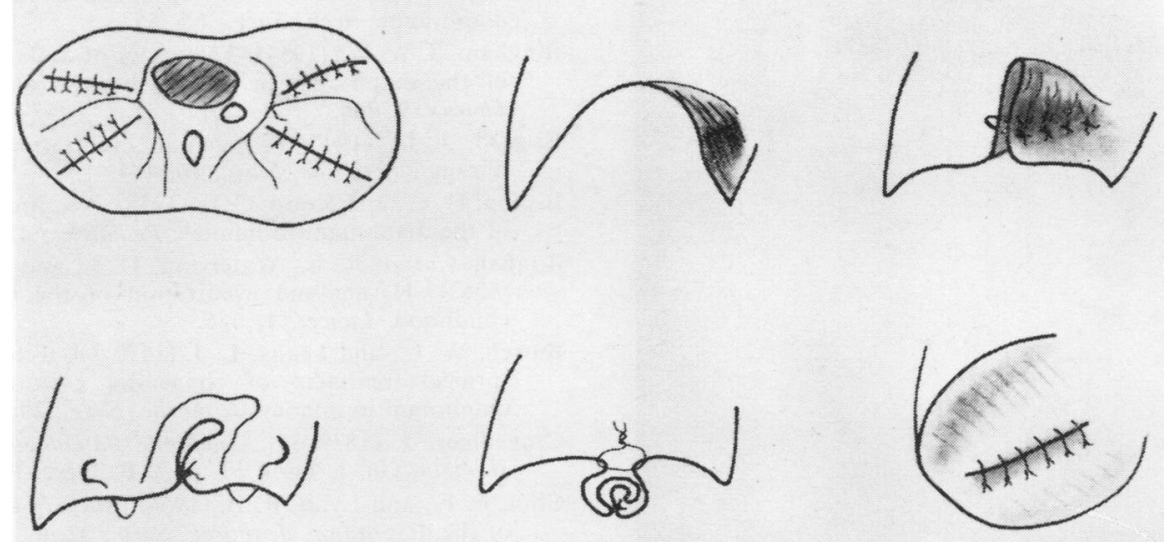

FIG. 3. Diagram showing our procedure: the diaphragm is pulled taut in a radial direction towards the mediastinum, held in place with a few stitches, and followed by multiple invaginating sutures.

Of the seven cases requiring surgery, three showed only respiratory symptoms consisting of repeated attacks of acute bronchopneumonia accompanied by dyspnoea and cyanosis. In one case there was anorexia and sporadic vomiting, and in two both respiratory and abdominal signs were present. In one case the child attended hospital only because of the chest wall deformity. The low incidence of digestive symptoms is due, in our opinion, to the predominance of rightsided lesions as well as to the difficulty of detecting subjective symptomatology such as postprandial heaviness, palpitations after feeding, or heartburn in infants. A marked underdevelopment was evident in the weight of cases 1, 2, and 6, and in a lesser degree in case 4 .

In eight cases there was bulging of the right hemithorax and a scaphoid abdomen, and in one of these the relaxation was more pronounced in the posterior portion, forming a posterolateral protuberance with an anterolateral depression of the chest.

The radiological picture was pronounced in seven of the nine infants, the dome reaching to the fourth to fifth intercostal spaces. The eventration was complete in all except case 9 in whom only the posterior part of the diaphragm was involved. Mediastinal movement on fluoroscopy appeared in six cases, and in five of these the eventrated diaphragm had the characteristic pattern of movement described by Chin and Lynn (1956).

As regards technique, we are in favour of a diaphragmatic plication performed by an intercostal approach (Fig. 3). To achieve this the diaphragm is stretched in a radial direction towards the mediastinum (Fig. 4) and a few mattress sutures of 1-0 Terylene are taken to hold the diaphragm in place. The suture line must always be parallel to the distribution of the phrenic nerve. We have never firmly fixed the plicated diaphragm to the wall of the thorax, as has been done by Quenu and Herlemont (1953) through the thorax, and by Butsch and Leahy (1950) through the abdomen, for if it is left firmly tied it is only at the expense of mobility. For this reason we prefer to invaginate the plication line with multiple sutures, taking the number of layers necessary in order to leave the diaphragm taut and lowered one or two intercostal spaces beyond the level that we ultimately wish to achieve.

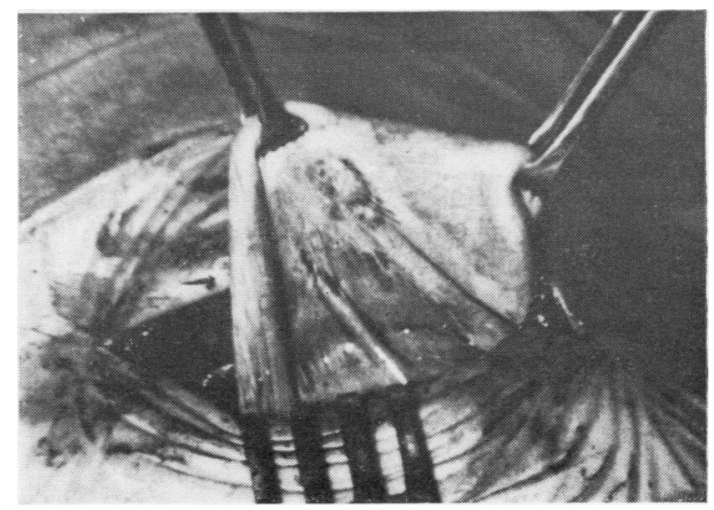

FIG. 4. Operative view. The muscle is tensed before beginning the plication. 


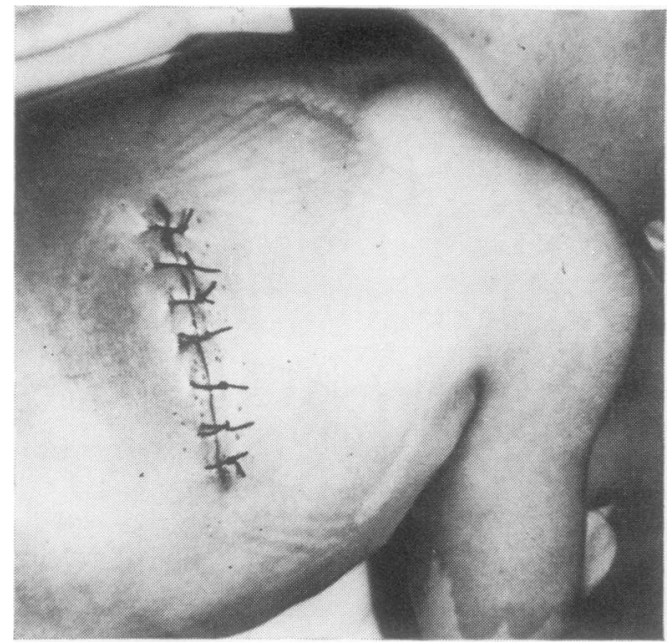

FIG. 5. Minimal posterolateral thoracotomy. Detail of the cutaneous incision.

Other techniques are those of incision followed by an overlapping imbrication suture or excision of the relaxed tissue (Bingham, 1954; Chin and Lynn, 1956 ; Bishop and Koop, 1958). These methods expose the patient to rupture of the suture line and secondary hernia.

We believe that surgery should be performed through the thorax, as it is easier to work over the vertex of a convexity than in the hollow of a concavity. However, it should not be forgotten that authors such as Butsch and Leahy (1950) and State (1949) have operated using the subcostal abdominal approach. Notwithstanding these methods, our results incline us towards the thoracic approach because this leads to fewer postoperative complications and an average hospital stay of five days. All operations were performed through a small thoracotomy (Fig. 5).

\section{REFERENCES}

Bayne-Jones, S. (1916). Eventration of the diaphragm, with report of a case of right-sided eventration. Arch. intern. Med., 17, 221.
Beck, W. C., and Motsay, D. S. (1952). Eventration of the diaphragm. Arch. Surg., 65, 557.

Bingham, J. A. W. (1954). Two cases of unilateral paralysi of the diaphragm in the newborn treated surgically Thorax, 9, 248.

Bisgard, J. D. (1947). Congenital eventration of the dia phragm. J. thorac. Surg., 16, 484.

Bishop, H. C., and Koop, C. E. (1958). Acquired eventration of the diaphragm in infancy. Pediatrics, 22, 1088.

Bonham-Carter, R. E., Waterston, D. J., and Aberdeen, E心 (1962). Hernia and eventration of the diaphragm in childhood. Lancet, 1, 656.

Butsch, W. L., and Leahy, L. J. (1950). A technique for the्ठ surgical treatment of congenital eventration of the diaphragm in infancy. J. thorac. Surg., 20, 968.

Cruveilhier, J. (1829-35). Anatomie Pathologique du Corpฎ Humain, vol. 1, book 17, p. 2. Baillière, Paris.

Chin, E. F., and Lynn, R. B. (1956). Surgery of eventrationof the diaphragm. J. thorac. Surg., 32, 6.

Christensen, P. (1959). Eventration of the diaphragm? Thorax, 14, 311.

Dor, J., Richelme, H., Aubert, J., and Boyer, R. (1969)

l'Eventration diaphragmatique. J. Chir. (Paris), 97, 399.

Jaubert de Beaujeu, M., and Mollard, P. (1965). Paralysiê obstétricale du diaphragme chez le nourrison. Indications et modalités du traitement chirurgical. Ann. ChirD thorac. Cardiovasc., 4, 469. (Ann. Chir.. 19, 973).

McNamara, J. J., Paulson, D. L., Urschel, H. C., an Razzuk, M. A. (1968). Eventration of the diaphragmo Surgery, 64, 1013.

Monereo, J., Cortes, L., and Dominguez, J. (1970). Hernias diafragmaticas del niño con análisis de las no hiatales y su mortalidad. Cirug. Española, 24, 135.

Petit, J. L. (1790). Traité des Maladies Chirurgicales, et de Opérations qui Leur conviennent, vol. 2, p. 233은 Méquignon, Paris.

Quenu, J., and Herlemont, P. (1953). Du traitement chirurgica de l'eventration diaphragmatique. J. Chir. (Paris), 69@્㐅 101.

Reed, J. A., and Borden, D. L. (1935). Eventration of the diaphragm; with a report of two cases. Arch. Surg., 31\% 30.

Richard, J., Chevalier, V., Capelle, R., Cavrot, E., Content, J., and Delforge, J. (1957). La paralysie diaphrag음 matique obstétricale. Arch. franç. Pédiat., 14, 563. , Henry, C., Pelc, S., and Content, J. (1959) L'eventration diaphragmatique chez l'enfant. Arch franç. Pédiat., 16, 300.

State, D. (1949). The surgical correction of congenita eventration of the diaphragm in infancy. Surgery, 250 461.

Thomas, T. V. (1970). Congenital eventration of the dia phragm. Ann. thorac. Surg., 10, 180. 